\title{
Corrigendum to "Utilization of Preventive Measures for Glucocorticoid-Induced Osteoporosis among Veterans with Inflammatory Bowel Disease"
}

\author{
Aikaterini Thanou, ${ }^{1}$ Tauseef Ali, ${ }^{2,3,4}$ Omar Haq, ${ }^{2,3}$ Sindhu Kaitha, ${ }^{2,3}$ Jordan Morton, ${ }^{3}$ \\ Stavros Stavrakis, ${ }^{3}$ and Mary Beth Humphrey ${ }^{3,4}$ \\ ${ }^{1}$ Arthritis and Clinical Immunology Research Program Oklahoma Medical Research Foundation, 825 NE 13th Street, MS 22, \\ Oklahoma City, OK 73104, USA \\ ${ }^{2}$ Inflammatory Bowel Disease Center, 825 NE 10th Street, Oklahoma City, OK 73014, USA \\ ${ }^{3}$ Department of Medicine, University of Oklahoma Health Sciences Center, P.O. Box 26901, WB1140, Oklahoma City, OK 73126, USA \\ ${ }^{4}$ Oklahoma City Veterans' Affairs Medical Center, 921 NE 13th Street, Oklahoma City, OK 73104, USA \\ Correspondence should be addressed to Aikaterini Thanou; aikaterini-thanou@omrf.org
}

Received 19 April 2017; Accepted 4 May 2017; Published 18 September 2017

Copyright (C) 2017 Aikaterini Thanou et al. This is an open access article distributed under the Creative Commons Attribution License, which permits unrestricted use, distribution, and reproduction in any medium, provided the original work is properly cited.

In the article titled "Utilization of Preventive Measures for Glucocorticoid-Induced Osteoporosis among Veterans with Inflammatory Bowel Disease" [1], there was an error regarding the FRAX ${ }^{\circledR}$ tool, which should be clarified as follows.

The article notes, "the WHO fracture risk predictor model (FRAX) designed to incorporate such factors in a comprehensive assessment tool has shown promising preliminary results in patients with IBD". However, the World Health Organization (WHO) did not develop, test, or endorse the FRAX tool or its recommendations [2]. The metabolic bone disease unit at the University of Sheffield that developed FRAX was a WHO Collaborating Centre from 1991 to 2010, but treatment guidelines must undergo a formal process before they can be endorsed by the WHO.

\section{References}

[1] A. Thanou, T. Ali, O. Haq et al., "Utilization of preventive measures for glucocorticoid-induced osteoporosis among veterans with inflammatory bowel disease," ISRN Gastroenterology, vol. 2013, Article ID 862312, 5 pages, 2013.

[2] Nathan Ford, Susan L. Norris, and Suzanne R. Hill, "Clarifying WHO's position on the FRAX ${ }^{\circledR}$ tool for fracture prediction," Bulletin of the World Health Organization, vol. 94, 862 pages, 2016. 


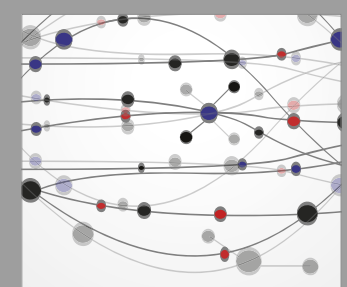

The Scientific World Journal
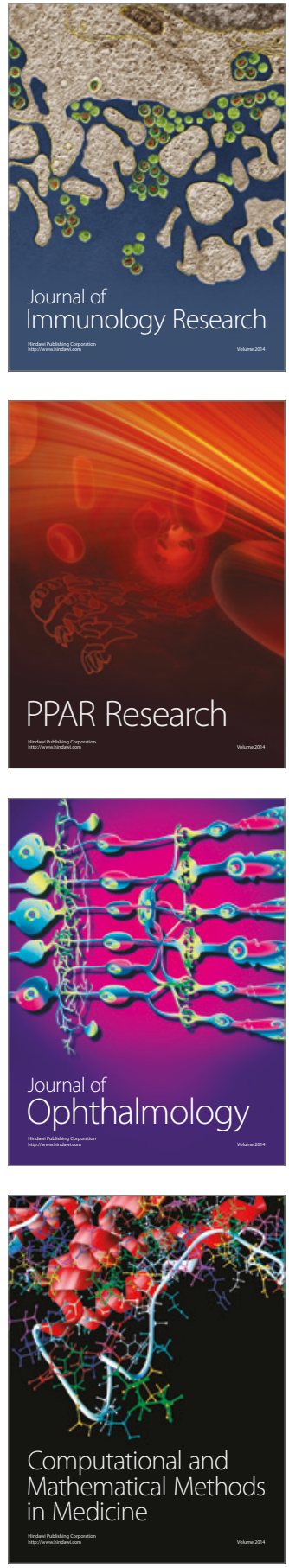

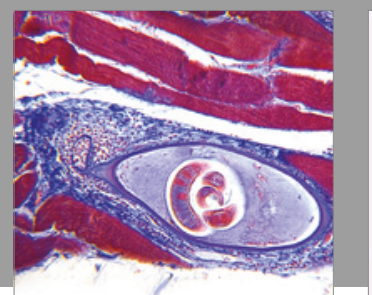

Gastroenterology Research and Practice
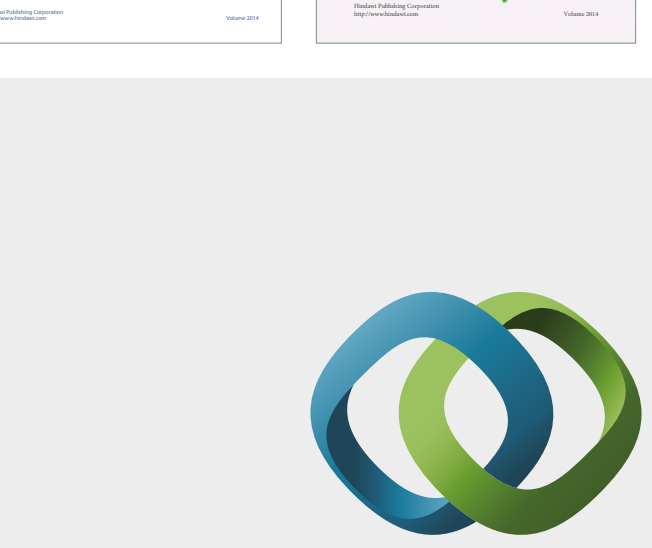

\section{Hindawi}

Submit your manuscripts at

https://www.hindawi.com
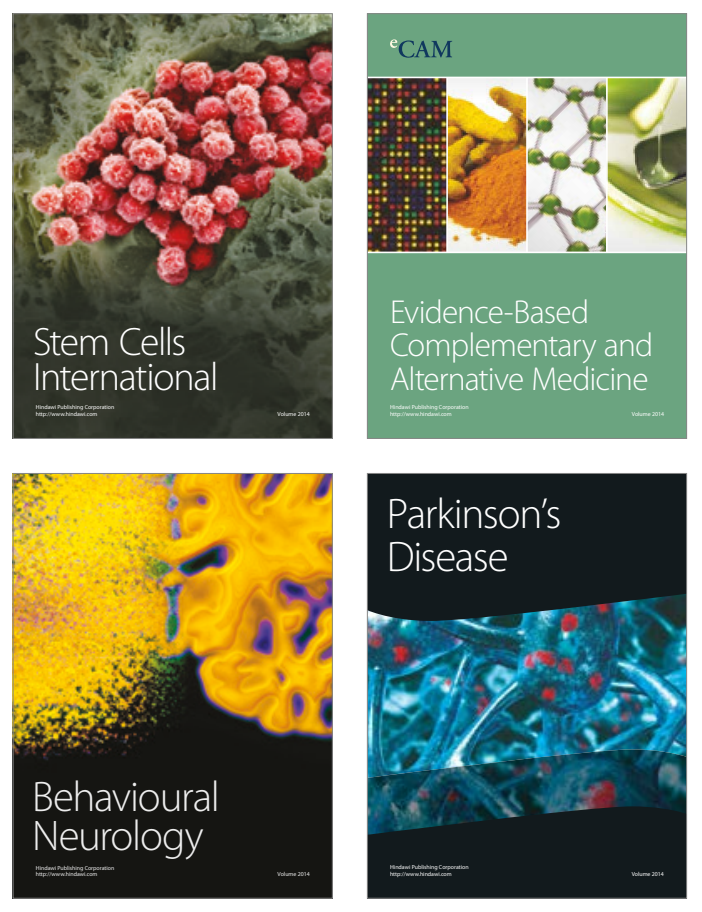
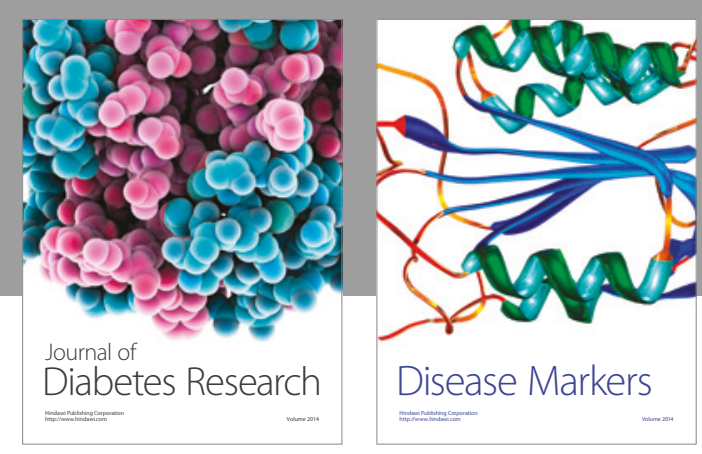

Disease Markers
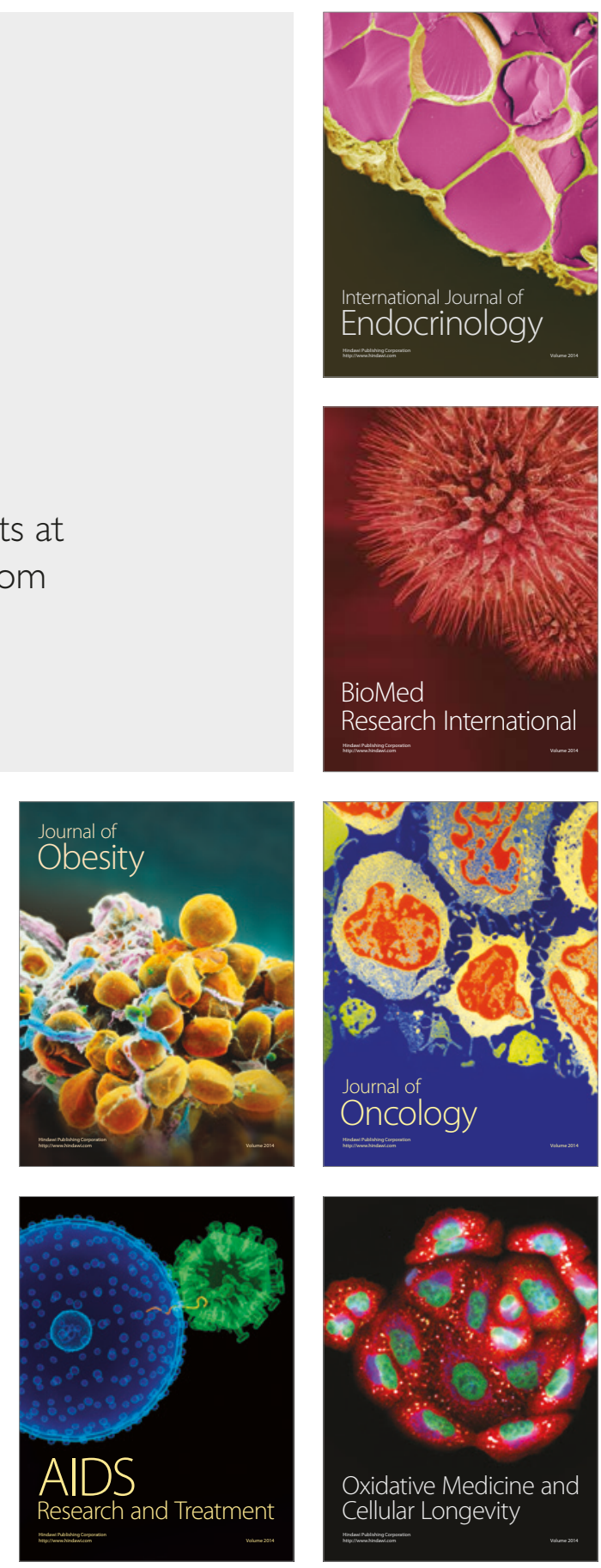